\title{
Trading wireless capacity through spectrum virtualization using LTE-A
}

\author{
Marcela M. Gomez \\ Liu Cui \\ mmg62@pitt.edu \\ lic49@pitt.edu \\ Martin B.H. Weiss \\ mbw@pitt.edu \\ School of Information Sciences \\ University of Pittsburgh \\ Pittsburgh, Pennsylvania 15260
}

\begin{abstract}
Markets for spectrum were first proposed by Ronald Coase [1] as a way to efficiently allocate this resource. It took another forty years for primary markets to be developed (in the form of spectrum auctions) as the mechanism for assigning spectrum licenses to users. It is not a secret that secondary markets would be necessary to fully realize the benefits of economic allocation of spectrum. But this is easier said than done, since spectrum is a complex, multi-dimensional product with relatively few buyers and sellers (at least for commercial mobile services), so liquid secondary markets have not emerged, even though spectrum trading through brokers is commonplace.

In this paper, we find that liquidity for spectrum markets can be improved over "naked" spectrum markets $[2,3]$ when a standardized commodity can be traded that uses the principles of spectrum virtualization [4]. We utilize the Physical Resource Blocks (PRBs) of LTE-Advanced as the traded commodity and modify the SPECTRAD model developed in [5] accordingly. Though much remains to be done, we find that this is a promising approach to finally realizing liquid secondary markets in radio spectrum.
\end{abstract}




\section{Introduction}

Liquid secondary markets for radio spectrum remain an elusive goal. Although license trading is fairly active in the US [6], transparent, liquid markets similar in nature to commodities exchanges are not. Previous research showed that simulated spectrum trading markets (called SPECTRAD)are not liquid when considering the features of today's mobile carrier industry [2]. Over the past several years, we have continued to analyze the feasibility of secondary markets for spectrum by first breaking some of the idealistic considerations built into SPECTRAD, which is described in detail in [5]. In particular, we modified the model to include the reality that spectrum is not perfectly fungible, which had an impact on the liquidity of markets. With this in mind, our goal in this paper is to find a scenario that would adapt "smoothly" to the multidimensionality characteristics of spectrum. By "smoothly" we mean that this constraint would become transparent to the users and does not increase transaction costs exponentially to the point of making markets inoperable.

With this in mind, we apply the concept of wireless networks virtualization based on LTE-Advanced features to explore how this results in a new trading commodity and adapts to existing notions of secondary markets for spectrum trading. Our overall goal is to determine whether these new considerations make markets thicker, which increase the users' willingness to participate without a major increase of costs and congestion. In other words, we wish to determine whether we could obtain viable, yet realizable secondary spectrum market by incorporating LTE-A virtualization concepts to our secondary spectrum market model, we obtain viable, yet realizable, markets.

\subsection{Background}

First of all, we would like to take a quick look back and remember some of the reasons that render the analysis of secondary markets for spectrum important.

\subsubsection{Spectrum Markets Overview}

From a general perspective, a secondary market can be defined as the market in which a seller of a good is not the one selling that good for the first 
time [6]. A primary market for spectrum was established in the 1990s with the adoption of spectrum auctions; nevertheless, the fixed spectrum allocation approach accompanying the primary market led to scarcity and other inefficiencies, which have driven researchers, industry members and regulators to seek for alternate solutions. The Federal Communications Commission considered the creation of secondary markets for spectrum in 2000. In one of its first Policy Statements regarding this matter, it is stated:

We believe that an expanded system of private sector markets will serve the public interest by creating new opportunities for increasing the communications capacity and efficiency of spectrum use by licensees. Such secondary market transactions will thereby complement the primary assignment function performed by the Commission through its spectrum auctions and licensing processes. While secondary markets are not a substitute for finding additional spectrum when needed and should not supplant our spectrum allocation process, a robust and effective secondary market for spectrum usage rights could help alleviate spectrum shortages by making unused or underutilized spectrum held by existing licensees more readily available to other users and uses and help to promote the development of new, spectrum efficient technologies". 1

Supporting the validity of secondary markets, Professor Peter Cramton suggested, "secondary markets are essential for the efficient and intensive use of spectrum. Secondary markets identify gains from trade that are unrealized by the primary market which in this case is the FCC spectrum auctions". ${ }^{2}$

The statements above portray the initial objectives supporting the development of secondary markets for spectrum. Further analyses perceive secondary markets as a means to ensure that, with changes in demand and supply, spectrum will migrate to more efficient uses, including those by parties not belonging to the initial allocation [6]. Note that embedded in all these considerations is one of the most important goals of secondary markets, which is to assign spectrum to users (and uses) who value it the most.

\footnotetext{
${ }^{1}$ Federal Communications Commission Policy Statement in the matter of Principles for Promoting the Efficient Use of Spectrum by Encouraging the Development of Secondary Markets. p.1.

${ }^{2}$ Professor Peter Cramton Statement at the Secondary Market Forum of the Federal Communications Commission. May 31, 2000.
} 
It is important also to mention that the prices set in the market have the ability to capture information regarding demand and supply in a manner that outperforms that of a centralized entity [7], reflecting the actual status of the interactions of buyers and sellers and, consequently, the real spectrum valuation.

Over time, we have come across several characteristics that secondary markets should have and the challenges these markets would encounter in their efficient development. In the FCC Policy Statement from December 2000, five essential elements for a market system to operate most effectively are mentioned:

- Clearly defined economic rights

- Full information on prices and products available to all participants

- Mechanisms for bringing buyers and sellers together to make transactions with a minimum of administrative cost and delay

- Easy entry and exit to the market by both buyers and sellers

- Effective competition, with many buyers and sellers.

In [8] the author mentions that the success of a secondary market for spectrum depends on the trading mechanism for minimizing transaction costs and maximizing the traders' surplus. Additionally, spectrum sellers and buyers should be given appropriate incentives for participating in the market (e.g., spectrum buyers obtaining spectrum to provide the same service as the sellers may reduce the willingness of the licensee to offer his spectrum for sale; spectrum buyers should be given guarantees of the availability of the spectrum they are acquiring). Further, in [9], Crocioni mentions several constraints that contribute to the delay in the deployment of secondary markets, among which we shall highlight the heterogeneity of spectrum, which might give rise to various secondary spectrum markets. Consequently, the information on the price paid for a specific frequency may not be indicative of the value of another frequency.

The aforementioned facts reinforce Professor Cramton's observation in the first FCC Forum for the discussion of secondary markets, where he envisioned that getting to the point in which spectrum becomes a true commodity in the marketplace will take some time and a lot of work due to the complexities inherent in radio spectrum. 
Even under the presence of important constraints and challenges, as in any other market type, in a secondary market for spectrum, trade will occur if a market entrant (or another incumbent) values a given spectrum portion more than the current incumbent and if the difference between the two valuations is larger than the transaction costs [9].

\subsubsection{The Appropriate Trading Commodity}

It is quite clear that our asset of interest, in this particular market, is electromagnetic spectrum. Nevertheless, when we think about electromagnetic spectrum, its variability and multidimensionality are the features that will mostly draw our attention. Various authors define a number of these dimensions; for instance, in [10] Matheson and Morris define seven dimensions in which spectrum can vary: frequency, time, three dimensions of location (latitude, longitude and elevation) and two dimensions of arrival (azimuth and elevation angles). For a market to be successful, we would need a "one-toone" match of supply and demand, or in other words, as Mayo and Wallsten express in [6], "Successful secondary market transfers require an alignment of the buyers' demands for spectrum of a particular dimension with the willingness of spectrum holders to supply spectrum in the same dimension". Under this conditions we would expect the resulting markets to be thin, yielding a lack of liquidity.

Spectrum trading scenarios have already been analyzed under the conditions mentioned above $[3,11]$, in which the trading commodity was considered to be "naked spectrum". In [11], electromagnetic spectrum was regarded as perfectly fungible, creating in this manner a completely homogeneous commodity. By incorporating fungibility limitations and constraints to the model, in [3], we could determine how this affected the trading choices and patterns of users and thus the final market viability result. In both cases, liquidity outcomes were reached; not surprisingly, those of the second case were fewer than those of the first, homogeneous case. However, as presented in [3], the market scenarios associated with liquidity in the second case are difficult to envision in reality (e.g., market liquidity involves the participation of a higher number of secondary users than the number of participants in the current telecommunications market). Thus, we believe that it is critical to determine a spectrum-related commodity that would permit us to maintain the market thickness obtained when trading a homogeneous commodity; in other words, a commodity that would bypass the spectrum heterogeneity 
constraints.

One possible approach to reach that goal would be to make some of the physical attributes of spectrum transparent to buyers and sellers and find a substitute commodity appealing to their needs. We find this achievable through wireless network virtualization.

\subsubsection{Wireless Networks Virtualization}

In a broad sense, virtualization refers to the creation of a virtual version of something, rather than the actual thing itself [12]. Nevertheless, when we think of network virtualization, we tend to more generally associate this concept with Computer Science. In [13], a thorough definition of this type of virtualization is provided: "Network virtualization is any form of partitioning or combining a set of network resources, and presenting (abstracting) it to users such that each user, through its set of partitioned or combined resources has a unique, separate view of the network. Resources can be fundamental (nodes, links) or derived (topologies), and can be virtualized recursively. Node and link virtualization involve resource partition/combination/abstraction; and topology virtualization involves new address spaces".

This concept maps quite well to the particular virtualization scope that we would like to focus on, which is Wireless Networks Virtualization. Within these networks, different components can be virtualized, and according to them, again, virtualization can take different shapes and nuances. As stated in [13], a given type of virtual network could become a different type of virtual network once we change the perspective. For instance, within the domain of wireless networks virtualization, we could explore infrastructure virtualization, air-interface virtualization or the virtualization of additional network components. Hence, even if at the basis we are dealing with the same network, by applying different types of virtualization, we will surely obtain distinct perspectives and thus achieve results specific to them.

One of the main reasons behind the development of wireless networks virtualization is to create such a network that could foster a greater amount of spectrum users, by means of creating further alternatives in terms of using, sharing, accessing and assigning the existing resources. For instance, through infrastructure virtualization and sharing, costs for new market entrants would be significantly reduced, thus increasing the incentives for new network operators to participate in the market. Additionally, a myriad of new services could be offered while using the same resources that were previously used 
by only one network operator. Further, as mentioned in [14], virtualization brings flexibility to the network; in other words, it gives the operators the required capabilities to expand or shrink their networks, according to their needs, without incurring in prohibitive costs.

Delving a little bit deeper into the virtualization of wireless networks, we can point out some interesting approaches presented in [4, 15-17]. In the aforementioned works, the resources belonging to the primary users of wireless networks are considered to be part of a pool, which is then made available, not to a single user, but to a large number of them. The notion of having a common pool of resources will, in turn, allow for a wider range of contributors to this pool, in addition to representing increasing incentives for investment in infrastructure [4]. It is also worth pointing out that a special focus on the restructuring of the network value chain ${ }^{3}$ is made. Indeed, the new value chain envisioned is aimed at providing the opportunity for parallel coexisting activities, the opportunity for a wider variety of participants, in addition to the opportunity for specialized as well as mainstream activities $[4]$.

In our work, we would like to emphasize this particular nuance of virtualization: the creation of a pool of resources, to which spectrum users can contribute, and from which they can benefit. It is clear that there is a large amount of (possible) resources pertaining to the pool; however, in this stage, we will focus our attention on the electromagnetic spectrum and its role within the pool. The specific technology that will accompany our wireless network virtualization analysis is LTE-Advanced. An overview of the advantages and constraints of this choice are presented in what follows.

\subsubsection{LTE-Advanced}

Long Term Evolution, LTE, originated as an standard whose main objectives were to minimize the system and user equipment complexities, allow a more flexible spectrum allocation in the existing or newly available frequency bands and to enable co-existence with current and legacy radio access technologies [18]. The migration from LTE to LTE-Advanced was driven by the incorporation of distinct features that would allow for the enhancement of the network and achievement of higher capacity. The most important

\footnotetext{
3 "The value chain includes all the activities that exist as a result of usage of the cellular network. The purpose of creating the chain is to understand where the costs are incurred and the revenue is generated" [4]
} 
new additions found in LTE-Advanced are the capability to perform carrier aggregation in order to achieve wider bandwidths; enhanced multi-antenna techniques in the uplink an downlink and support for the use of relay nodes, which connect to relay-enabled eNBs, thus improving throughput. ${ }^{4}$

In LTE, the Physical Resource Block (PRB) is the basic element for radio resource allocation. A PRB is a set of resource elements, which are timefrequency resource-units for uplink and downlink transmission. Resource elements can be defined as one sub-carrier over one OFDM symbol. In total, 12 OFDM subcarriers, contiguous in frequency, over one slot in time will form a PRB. Summing up, the time-frequency region that encloses a PRB corresponds to a 0.5 millisecond-time slot and $180 \mathrm{kHz}$ in the frequency domain (12 subcarriers x $15 \mathrm{kHz}$ each) [18, 19].

The minimum size of radio resource that can be allocated is the minimum Time Transmission Interval (TTI) in the time domain, which corresponds to one subframe of 1 millisecond, which in turn corresponds to two resource blocks. Subframes can be further grouped into frames of 10 milliseconds length with specific arrangements of the PRBs for FDD and TDD transmission.

The number of allocated PRBs will contribute to the bandwidth a specific user can count on for given transmission; nevertheless, the actual number of PRBs that users can be allocated is determined by the standard and is presented in the following table. Additionally, Table 1 provides information on further details associated to the allocation of the PRBs, such as the required guardband, the actual occupied bandwidth and the number of subcarriers involved in the transmission $[19,20]$

\begin{tabular}{rcccccc}
\hline LTE Parameters for Downlink Transmission \\
\hline Number of Resource blocks & 6 & 15 & 25 & 50 & 75 & 100 \\
Number of Occupied Subcarriers & 72 & 180 & 300 & 600 & 900 & 1200 \\
Transmission Bandwidth [MHz] & 1.4 & 3 & 5 & 10 & 15 & 20 \\
Occupied Bandwidth [MHz] & 1.1 & 2.7 & 4.5 & 9.0 & 13.5 & 18.0 \\
Guardband [MHz] & 0.32 & 0.3 & 0.5 & 1.0 & 1.5 & 2.0 \\
\hline
\end{tabular}

Table 1: LTE Parameters for Downlink Transmission

Using the carrier aggregation capabilities of LTE-A, when wider band-

\footnotetext{
${ }^{4}$ http://www.3gpp.org/technologies/keywords-acronyms/97-lte-advanced
} 
widths are required, these can be defined in contiguous and non-contiguous spectrum deployments and can sum up to $100 \mathrm{MHz}$. These bandwidths can be achieved through the aggregation of up to five component carriers. The individual component carriers could have different bandwidths and the number of aggregated carriers could be different in uplink and downlink; however, the number of uplink component carriers cannot be larger than the number of downlink component carriers. ${ }^{5}$ Due to the discontinuous nature of the spectrum that has been reserved for this technology, the bandwidth available is rather fragmented. Hence, the user terminals should have filtering, processing and decoding capabilities for this large and variable bandwidth. This certainly increases the complexity of the user terminals and is one of the major challenges contemplated by the LTE standard $[18,21]$

As can be observed in Table 1, there is a specific and direct mapping between the number of PRBs and transmission bandwidth. In this paper, we plan to make use of this mapping to manage the spectrum-related commodity available within the pool of resources. In other words, we would like the pool of resources to be composed of a certain number of PRBs which in turn correspond to specific values of bandwidth that can be further translated into capacity rates. Note that by applying the carrier aggregation concepts and features, we can merge resources from the pool in order to obtain greater bandwidths and thus higher capacity values. As expected, this is the point in which the actual physical electromagnetic frequency becomes transparent to the users, and they are asked to deal with an additional, perhaps more manageable feature, which is capacity.

It is worth mentioning that another important reason to consider LTE-A for our analysis is that spectrum users will be utilizing devices compatible with this standard. Thus, we are not concerned about the capabilities of the equipment antennas to tune to the range of frequencies pertaining to this standard (at least within a given country), even if this range is discontinuous.

\section{Methods}

As mentioned in section 1.1.3, if we narrow down the scope of virtualization one step further and adapt it to this paper, we can study the specifics of one of the resources pertaining to the aforementioned pool: spectrum. As part of the pool, spectrum will be accessible to a larger number of network

\footnotetext{
${ }^{5}$ http://www.3gpp.org/technologies/keywords-acronyms/97-lte-advanced
} 
operators, instead of being available only to the incumbent. The distribution of the spectrum resources from the pool could be well performed via spectrum markets as stated in $[4,17]$, which is an approach that could well fit with our secondary markets for spectrum trading framework. Nonetheless, we shall remember that licensed spectrum has long been considered as the bottleneck of the modern-day wireless networks and as the particular resource for which there are no substitutes in the network [17]. However, even if there are not actual physical substitutes for "naked spectrum", we could adapt additional notions of wireless virtualization in order to make this resource more readily available and create the illusion of infinite resources that is needed to fulfill the spectrum demands [4], which may also represent the enhancement of liquidity of secondary markets for spectrum that we are looking for.

By appealing to spectrum virtualization, then, we switch focus from constituting a pool of electromagnetic frequencies to a pool of spectrum-related commodities that would be more easily manageable in the market. By this we mean, commodities whose underlying frequencies would be transparent to the users accessing the market, thus giving them further opportunities and taking away the constraints related to the physical frequencies incompatibilities.

Our choice of interest, regarding the appropriate market commodity, would be to contemplate the trading of wireless capacity. In such a scenario, users would bid for specific amounts of capacity that they would need to fulfill their traffic requirements without being concerned about which are the actual electromagnetic frequencies that are available in the market. A central entity (i.e., Band Manager) would be in charge of performing the appropriate mapping of the capacity required by the buyers with the capacity available in its inventories, which in turn belongs to the pool of spectrum resources.

Even though we are making physical frequencies transparent in the market through virtualization, we shall still consider the technical feasibility of this approach. For example, an important constraint to consider is that devices are technically limited in their capabilities to tune to a wide range of frequencies. So, as a first step toward the analysis of the viability of this method and the actual impact in the market liquidity, we propose that the frequencies belonging to a specific technology, such as LTE-A, should be considered. Hence, we can assume that all the users involved in the trade are capable of tuning to all the physical frequencies associated with the capacityoffers that are available in the market. This is not a limiting assumption since 
end user devices will all (eventually) have this capability anyway.

In conclusion, we can summarize the concepts and ideas we have explored so far as follows: The management and distribution of the resources pertaining to the pool will be in charge of the secondary markets for spectrum trading via auction methods. The specific reason why we are appealing to virtualization is to enhance the market transactions and hopefully bring more liquidity to the market, given that at this instance, our objective is to make markets thicker. This is done by making the electromagnetic frequency transparent to the users when we switch the trading commodity from "naked spectrum" to a more manageable one, such as wireless capacity. In such a trading environment, users should only be concerned about bidding for the required amounts of capacity to fulfill traffic requirements, irrespective of the underlying electromagnetic frequency. At this specific point, we are ensuring the physical feasibility of our proposal given that our work is framed within the LTE-A standard.

For this to render a successful market, users should have the appropriate guarantees about the commodities they are acquiring and thus the incentives to enter and remain active in the market (i.e., markets should be safe). These would be the specific perspectives of virtualization that we would like to incorporate into our secondary markets for spectrum trading framework and the benefits we desire to obtain from this fusion.

\subsection{Model Details}

In order to test the viability of the incorporation of virtualization concepts to secondary markets for spectrum trading, we have adapted our new structure to the existing spectrum trading agent-based model presented in [11]: SPECTRAD. In what follows, we will provide details regarding the market structure considered, the market participants, the commodities available in the market and the market transactions (e.g., the bidding and spectrum assignment process. So that our results might be comparable with the previously published results, we have kept the essential aspects of SPECTRAD intact.

\subsubsection{Market Structure}

The market type considered in our model is a Band Manager Exchangebased market. In general terms, this type of market model will have similar 
characteristics as those of the Band Manager Exchange presented in [11]; the Spectrum Exchange is the central entity of the market and its Band Manager functionality implies that the Exchange holds a spectrum inventory from which it has to assign leases to the spectrum requesters in the market. In this paper, the spectrum inventory that the Exchange holds corresponds to the previously mentioned spectrum pool. Additionally, when performing the spectrum-lease assignment process, the Band Manager will have to take into account the specific needs of the spectrum users without being oblivious to the requirements of the LTE-A standard. The two most important tasks that the Band Manager needs to consider is the assignment of PRBs in sets allowed by the standard (see Table 1) and the performance of carrier aggregation to provide for higher capacity requirements.

\subsubsection{Market Participants}

In the Band Manager Exchange-based markets, the spectrum users will be license requesters who are seeking spectrum in the market to fulfill their traffic obligations. Here, we envision these license requesters as Mobile Virtual Network Operators (MVNOs) or spectrum resellers who will be in charge of providing the spectrum acquired in the secondary market to their final customers.

Spectrum requesters can belong to one of three different levels of valuation of the spectrum: high, medium or low, according to the type of service they will provide. Along this lines, the level of spectrum valuation a given user has, will be directly proportional to his capacity requirements and to the stringency of the quality of service he needs to comply with. It follows that the price that the spectrum requester is willing to pay will be consistent with his own valuation of the spectrum.

The specific capacity requirements of each spectrum user, within the service area, are modeled as an exponentially distributed aggregate traffic demand with a mean of $4.0 \mathrm{Mbps}$. The interval between changes of traffic demand is modeled with an exponential distribution as well, with a mean value that is uniformly distributed between 10 and 25 simulation-time units.

\subsubsection{Trading Commodities}

The core of the analysis that we have performed is to develop a commodity

that could improve the liquidity of the secondary markets for spectrum. In 
this light, we modified the existing spectrum units or bandwidth units proposed in the original model of SPECTRAD [11], and we incorporated a new spectrum unit, which is compliant with the technology that we have chosen, which is LTE-A. The new units of spectrum sold in the market are Physical Resource Blocks or PRBs.

The number of resource blocks that are available in the market correspond to the pool inventory (i.e., the number of PRBs that the primary users of spectrum have decided to provide for trade in the pool); however, secondary user can be assigned PRBs only according to what the LTE-A standard dictates, which is, according to the allowable number of PRBs. In our model for resource availability, we have considered 3 bands of $10 \mathrm{MHz}$ each in the 700 $\mathrm{MHz}$ range, which, approximately, correspond to the bands $13(746 \mathrm{MHz}-756$ $\mathrm{MHz}), 14(758 \mathrm{MHz}-768 \mathrm{MHz})$ and $17(734 \mathrm{MHz}-746 \mathrm{MHz})$. These bands have been made available for LTE in the United States. The reason we have chosen these bands is because they provide relatively fungible transmission parameters due to their range similarity.

The bandwidth or size of the PRBs is determined according to the LTEA standard (see Table 1). Note that the actual value that we have used for our calculations is that of the Occupied Bandwidth, which corresponds to the Transmission Bandwidth - Guardband. In order to determine the traffic capacity of the PRBs, we have calculated the actual capacity that could be obtained with the bandwidth available to the highest frequency belonging to the available bands (so that we could portray the worst case scenario) using the Shannon Capacity formula (1). Where the signal corresponds to the power received at $1 \mathrm{Km}$ from the receiver with the considered frequency using COST231 Walfish-Ikegami Model. The noise is calculated using equation (2), where $\mathrm{F}$ is the noise figure, $\mathrm{K}$ is the Boltzmann constant, $T_{o}$ is the noise temperature and $\mathrm{B}$ is the considered bandwidth value.

$$
\begin{gathered}
C=B \log _{2}\left(1+\frac{S}{N}\right) \\
N=F K T_{o} B
\end{gathered}
$$

In the original SPECTRAD model, secondary users had the choice of opting for "alternate technologies" in case the spectrum price in the auction was above their price limit or in case spectrum was scarce in the market. Instead, we have linked the notion of alternate technologies directly to any type of unlicensed spectrum (e.g., TV Whitespaces, IEEE 802.11) that the 
spectrum requesters could opt for, when the PRBs in the market are not accessible. Note that spectrum requesters need to bear in mind that the quality of service they will receive with unlicensed spectrum is lower than that of licensed spectrum; hence, users valuation of licensed spectrum will be inversely proportional to their valuation of the alternate, unlicensed spectrum option. In this particular work, we have chosen TV Whitespaces in the 700 $\mathrm{MHz}$ band so that we remain in a close range to the LTE bands pertaining to the core of our analysis. Note that this assumption does not limit our choices for additional unlicensed bands, given that nowadays, most devices are in fact compatible with a large number of these frequencies.

In order to keep consistency with the basic LTE units, and considering that one PRB has a bandwidth of $180 \mathrm{KHz}$, we have considered this same basic unit for calculating the transmission capacity of unlicensed spectrum in our model. In this way, one unit of unlicensed spectrum will provide a level of capacity equivalent to the amount that can be achieved with $180 \mathrm{KHz}$ of unlicensed spectrum operating in the $700 \mathrm{MHz}$ band.

\subsubsection{Market Transactions}

We follow the assumption made in [11], which considers that the mechanism for matching buyers and sellers in the spectrum exchange is continuous double auctions. In this particular setting, we will be assigning the spectrum in the pool (provided by the spectrum licensees) to the spectrum requesters participating in the market.

In each bidding round, spectrum users will calculate their required amount of PRBs according to the traffic they have to meet. Based on their needs and on the maximum price they are willing to pay, users will post a bid in the current bidding round. At the end of each bidding round, the exchange will organize the bids according to price. If the demand is greater than the supply, the exchange will calculate the cutoff price according to the number of PRBs it can assign based on the LTE standard; otherwise, the cutoff price will be the minimum cutoff price set by the exchange, which has been set to 50.00 monetary units. After each bidding round, the cutoff price will be announced to the users, and they will decide to adjust their bids accordingly. Several bidding rounds will be conducted until the variation of the cutoff price is less than $0.5 \%$ from one round to the following or until a (preset) maximum number of bidding rounds is reached.

Once the bidding rounds have concluded, the spectrum assignment pro- 
cess takes place. It is performed by the exchange according to the LTE-A standard parameters and making use of carrier aggregation capabilities. In this way, the exchange will calculate the number of resource blocks to assign to the spectrum requesters taking into account the resources that it has in inventory and the possibilities that the standard provides for the aggregation. After the assignment process, the spectrum requesters will compare the amount of capacity they can meet with the resources received and the actual required capacity, and in case the resources received from the exchange are not enough, they will decide to use extra unlicensed spectrum units.

The PRBs' lease lasts 10 simulation-time units, which we map to the duration of an LTE frame, which is known to be 10 milliseconds. Once the 10 millisecond-period is over, the previously assigned PRBs return to the exchange inventory.

As we mentioned at the beginning of this subsection, we have been concerned about keeping the essential aspects of SPECTRAD intact in order to retain a constant basis for comparability. In this light, for our analysis, we have considered two cases regarding the duration of the unlicensed spectrum usage period. The first case exactly maps the duration of the "Alternate Technology units" (the alternative to licensed spectrum in the original model) to the duration of the unlicensed spectrum usage period; hence, a spectrum user will hold the bandwidth units of unlicensed spectrum for a period that will be uniformly distributed between 90 and 110 simulation time units. In the second case, we have diverged from this setting and we have assumed that the usage of unlicensed spectrum will last as long as the licensed spectrum lease (i.e., 10 milliseconds). This interpretation will permit us to simulate possible degradation in the service due to the large number of users sharing unlicensed spectrum and, perhaps more importantly, the fact that users would be allowed to enter the market one more time and analyze whether the conditions are profitable, granting them access to licensed PRBs.

In each of the two cases, users will enter the new set of bidding rounds to acquire a new licensed spectrum lease according to their current unlicensed spectrum holdings and traffic requirements.

The market transactions will last 5000 simulation time units (time ticks), from which 3000 are considered as warm-up period and the last 2000 will provide the data that will be analyzed and presented in our results section.

Table 2 presents a summary of important parameters relevant to the SPECTRAD model presented in this section. 


\section{General Model Parameters}

\begin{tabular}{|c|c|}
\hline $\begin{array}{l}\text { Bandwidth (occupied) of } \\
\text { the PRBs [MHz] }\end{array}$ & $\begin{array}{l}\text { Values permitted by the standard: } \\
{[1.08,2.7,4.5,9,13.5,18] \text {. }} \\
\text { Using carrier aggregation we can ob- } \\
\text { tain up to } 54 \mathrm{MHz} \text {. }\end{array}$ \\
\hline $\begin{array}{l}\text { Traffic capacity of a PRB } \\
\text { [Mbps] }\end{array}$ & $\begin{array}{l}\text { Calculated according to the Band- } \\
\text { width associated to the set of PRBs } \\
\text { assigned, using the Shannon Capac- } \\
\text { ity formula. } \\
\text { Min }=4.06 \mathrm{Mbps}, \mathrm{Max}=15.5 \mathrm{Mbps}\end{array}$ \\
\hline $\begin{array}{l}\text { Trading capacity of an Un- } \\
\text { licensed Transmission Unit } \\
\text { [Mbps] }\end{array}$ & $\begin{array}{l}\text { - Capacity calculated for } \\
\text { of bandwidth with the } 700 \\
\text { l }\end{array}$ \\
\hline PRBs lease time & cks (simulation time units) \\
\hline $\begin{array}{l}\text { Unlicensed spectrum usage } \\
\text { time }\end{array}$ & $\begin{array}{l}\text { Case 1: Uniformly distributed be- } \\
\text { tween } 90 \text { and } 110 \text { time ticks. } \\
\text { Case 2: } 10 \text { time ticks (simulation } \\
\text { time units) }\end{array}$ \\
\hline $\begin{array}{l}\text { Total simulated market } \\
\text { time }\end{array}$ & $\begin{array}{l}5000 \text { time ticks: } 3000 \text { for warm-up } \\
\text { period and } 2000 \text { for active data col- } \\
\text { lection to determine the market be- } \\
\text { havior }\end{array}$ \\
\hline \multicolumn{2}{|c|}{ Spectrum User Parameters } \\
\hline Number of Spectrr & $6,10,20$ \\
\hline Mean traffic Demand & $4.0 \mathrm{Mbps}$ \\
\hline $\begin{array}{l}\text { Mean Traffic Interarrival } \\
\text { Time }\end{array}$ & $\begin{array}{l}\text { Uniformly distributed between } 10 \\
\text { and } 25 \text { time ticks }\end{array}$ \\
\hline
\end{tabular}

Table 2: SPECTRAD Model Parameters

\section{Results}

The parameters and details presented in the previous section define the two scenarios that we simulated in order to evaluate our proposal. In a first instance, we considered the model in which the duration of the unlicensed spectrum usage was exactly the same as that of the lifetime of the originally used alternate technology units. Our goal in this scenario was to measure the 
impact of virtualization, itself, in the final market liquidity outcome. After evaluating these results, we proceeded to refine certain details that seem more appropriate to the actual behavior and usage of unlicensed spectrum (which is the alternative to licensed spectrum that we are considering in our current study). In this way, we would be able to determine if there is further impact on the resulting market viability, and how this compares to the original conditions of the SPECTRAD model.

In what follows, we will start by detailing the parameters and metrics that we have considered for the market viability evaluation in subsection 3.1. To conclude this section, we will present the actual market viability results obtained in each of our two scenarios in subsection 3.2 .

\subsection{Market Viability Score}

The market viability score was developed in [11] as a means to develop a quantifiable measure of how feasible it was for a given market to succeed. This score is based in the following five criteria which are determinant characteristics for the viability of a Band Manager Exchange Based-market:

- Probability of an empty bid list(\%): As markets progress, market participants whose bidding price is not competitive enough at the end of the bidding rounds will opt for unlicensed spectrum. It may be the case that these users will accumulate enough unlicensed spectrum units that will make them likely to opt out from the upcoming licensed spectrum bidding process. If all spectrum users reach this situation, the bid list received by the Band Manager will be empty, which is an undesirable condition for the market. The probability of an empty bid list corresponds to the average probability that in a given period of time there are no spectrum users making any bids for spectrum. A high value of this factor represents of lack of activity in the market [11].

- Probability that demand is greater than supply(\%): This parameter represents the probability that at a given time period, the spectrum requests surpass the Band Manager spectrum holdings.

- Average cutoff price: The minimum cutoff price set in our model is 50 monetary units. The higher the cutoff price in a bidding round, higher the valuation of the spectrum in that specific round. Additionally, this indicates that there was a significant level of market activity. 
On the other hand, when the cutoff price is exactly the minimum, it indicates that the demand is not greater than the supply, and thus, the spectrum price is not being set by the users' activity in the market.

- Average number of assigned Resource Blocks(\%): This parameter corresponds to the ratio of the amount of RBs that have been assigned in the market and the RBs that were available in the pool and controlled by the Band Manager. This metric gives us a notion of the level of efficiency achieved in the assignment of the spectrum via our market model.

- Average number of Unlicensed Spectrum units per spectrum user: In our model, a spectrum user would normally need 4 bandwidth units to fulfill his average traffic requirements. In this way, when users hold a larger amount of unlicensed bandwidth units, it indicates that they are utilizing only unlicensed spectrum to provide their services (without making use of licensed spectrum). This factor is in turn associated with the degree of ease or difficulty to obtain spectrum from the market.

Following the description above, and the guidelines provided in [11], Table 3 shows the viability criteria and the actual thresholds that have been considered for evaluation and final determination of market liquidity.

\begin{tabular}{|l|c|c|c|}
\hline \multicolumn{1}{|c|}{ Factor } & Pass & Fail & $\begin{array}{c}\text { Score } \\
\text { Pass } / \text { Fail }\end{array}$ \\
\hline Probability of empty bid list & $=0$ & $>0$ & $1 /-1$ \\
\hline $\begin{array}{l}\text { Probability that demand is } \\
\text { greater than supply }\end{array}$ & $\geq 10 \%$ & $<1 \%$ & $1 /-1$ \\
\hline Average cutoff price & $\begin{array}{c}\geq \text { Average } \\
\text { across all } \\
\text { markets }\end{array}$ & $\begin{array}{c}<\text { Average } \\
\text { across all } \\
\text { markets }\end{array}$ & $1 /-1$ \\
\hline $\begin{array}{l}\text { Percentage of assigned } \\
\text { spectrum }\end{array}$ & N/A & $\geq 4$ & $0 /-1$ \\
\hline $\begin{array}{l}\text { Average number of unlicensed } \\
\text { spectrum units per SU }\end{array}$ & & & \\
\hline
\end{tabular}

Table 3: Evaluation Criteria for the Viability of the Simulated Spectrum Trading Markets 
According to the conditions presented in table 3 and the data we obtained in our simulations, we detail the results that we have obtained for each of our simulation scenarios in the subsection that follows.

\subsection{Simulation Results}

The figures presented in each scenario correspond to the average values of 100 simulation runs for each combination of parameters applicable to our model details. More specifically, given that we have considered that the maximum availability of LTE resources would correspond to three $10 \mathrm{MHz}$ bands in the $700 \mathrm{MHz}$ range, this provides us with a spectrum pool that could have a minimum of $18 \mathrm{RBs}$ and a maximum of $150 \mathrm{RBs}$. We have constructed our simulation scheme following the same approach as in [11]. Thus, we utilized the variable " $\mathrm{R}$ " as a means to determine the number of RBs available in the market, as explained through the expression in (3).

$$
\text { number of } \mathrm{RBs}=\text { number of } \mathrm{SUs} \times R
$$

For each of our two simulation scenarios, we have considered different combinations of spectrum users and RBs available following the values of $\mathrm{R}$ presented in Table 4. Note that for the availability of RBs, we have considered values that could be aggregated through the addition of allowable quantities of RBs from the three $700 \mathrm{MHz}$ bands. In case the exact value was not achievable, we used the closest (allowed and higher) amount of RBs for our simulation; for example, in the case of 4 spectrum users and $\mathrm{R}=$ 5 , we would need a pool of $20 \mathrm{RBs}$ in our simulation. We could not use a standard-compliant amount of RBs from the 3 bands to obtain this value. Instead, we used instead a pool of $21 \mathrm{RBs}$, which results from aggregating $15 \mathrm{RBs}$ from one band and $6 \mathrm{RBs}$ from another. In the same way, we have limited our simulated combinations to those resulting in values $\leq 150$, hence, our simulated scenarios for 10 spectrum users end at $\mathrm{R}=15$, and in the case of 20 spectrum users, we consider only $\mathrm{R}=5$.

Under these circumstances, for both of the scenarios we have analyzed, we present the results particular to each of the market viability evaluation criteria we mentioned in subsection 3.1, and we finally include the resulting viability score that has been obtained in what follows. 


\begin{tabular}{|c|c|}
\hline Parameter & Value \\
\hline \hline Number of SUs & $4,5,6,10,20$ \\
\hline $\mathbf{R}$ & $5,10,15,20,15$ \\
\hline Number of RBs & Number of SUs $\times \mathrm{R}$ \\
\hline
\end{tabular}

Table 4: Combination of Parameters for Simulation Scenarios

\subsubsection{Scenario 1}

We tested our first, conservative approach, in order to remain as close to the original model as possible, and still be able to incorporate the expected notions of virtualization and technology compliance details (i.e., LTE-A standard parameters). In this subsection, we show the results of this first tested scenario. We start by showing how the model performed regarding each of the evaluated viability criteria in Figures $1-5$, and we finally aggregate our results in the market viability scores, which can be observed in figure 6 .

\section{Probability of Empty Bid List}

As spectrum scarcity diminishes in the market, the probability that there will be an empty bid list decreases as well. Under these circumstances, spectrum users have more access to the spectrum, thus, they are less likely to accumulate unlicensed spectrum units, which is the main cause for the lack of bidding activity. The results we present in figure 1 are consistent with the explanation above. We can see that in situations where there is not enough spectrum $(\mathrm{R}=5)$ and we have only a few spectrum users $(\mathrm{SUs}=4)$, the probability of having an empty bid list is equal to $4.8 \%$, whereas in situations of spectrum oversupply $(\mathrm{R}=25)$ and with a large number of market participants $(\mathrm{SUs}=20)$, the probability of having an empty bid list drops to $0 \%$. 


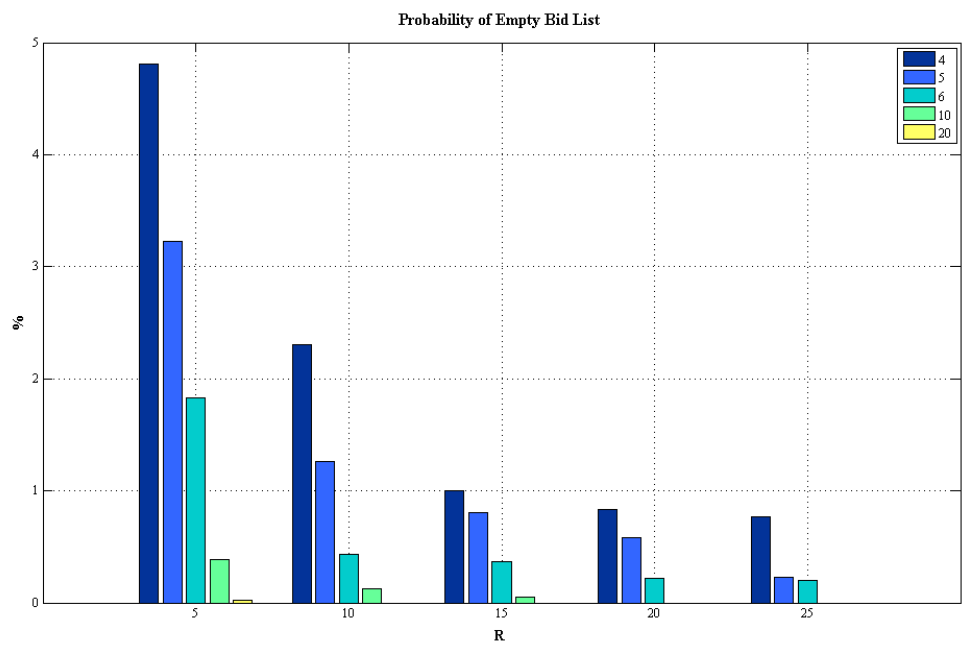

Figure 1: Probability that bid list is empty in Scenario 1

\section{Probability that demand is greater than supply}

In the case of demand and supply, as the provision of spectrum in the market increases, the value of this factor drops. In the same fashion, greater the number of market participants, greater the probability that spectrum demand in the market will surpass the Band Manager's spectrum inventory. In this particular scenario, we observe that the best conditions for this parameter are those of spectrum scarcity, when $\mathrm{R}=5$ and there are 20 spectrum users, with a probability of Demand being greater than supply of $58.8 \%$, while the worst case we encountered presented a level of $8.4 \%$, when $\mathrm{R}=25$ and we have only 4 spectrum users in the market. Figure 2 shows all the values pertaining for the different cases we tested in this simulated scenario. 


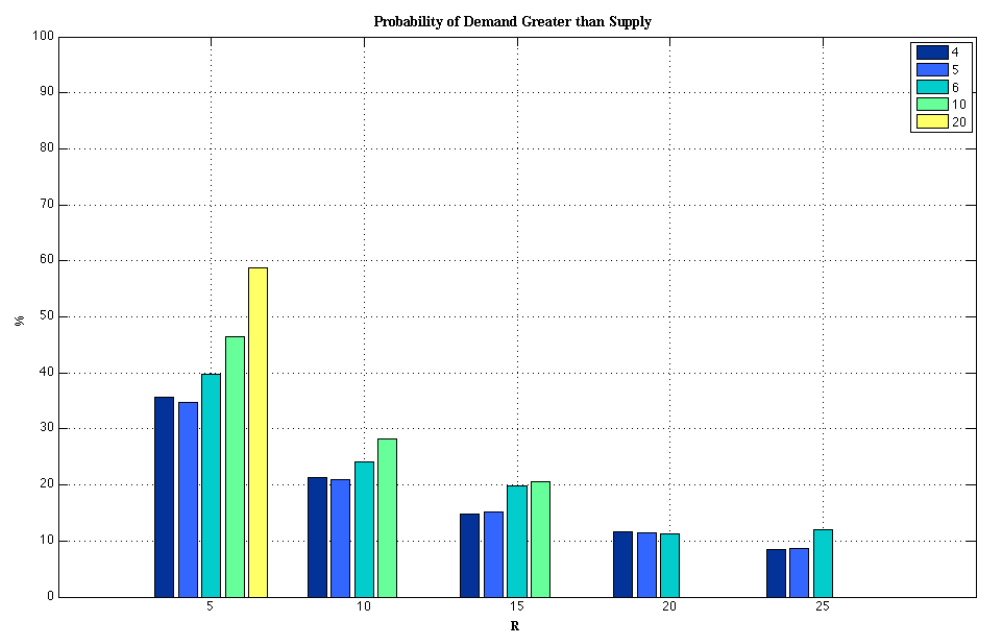

Figure 2: Probability that demand is greater than supply results for Scenario 1

\section{Average cutoff price}

In cases where there is spectrum scarcity in the market (i.e., $\mathrm{R}=5$ ) the price paid to obtain this desired commodity will be considerably high, especially when compared to situations of spectrum oversupply. This trend can be corroborated in figure 3, where we can find an average cutoff price as high as 108.1 monetary units when $\mathrm{R}=5$ and $\mathrm{SUs}_{\mathrm{s}}=20$, and this price can drop to 57.6 when $\mathrm{R}=25$ and $\mathrm{SUs}=4$. 


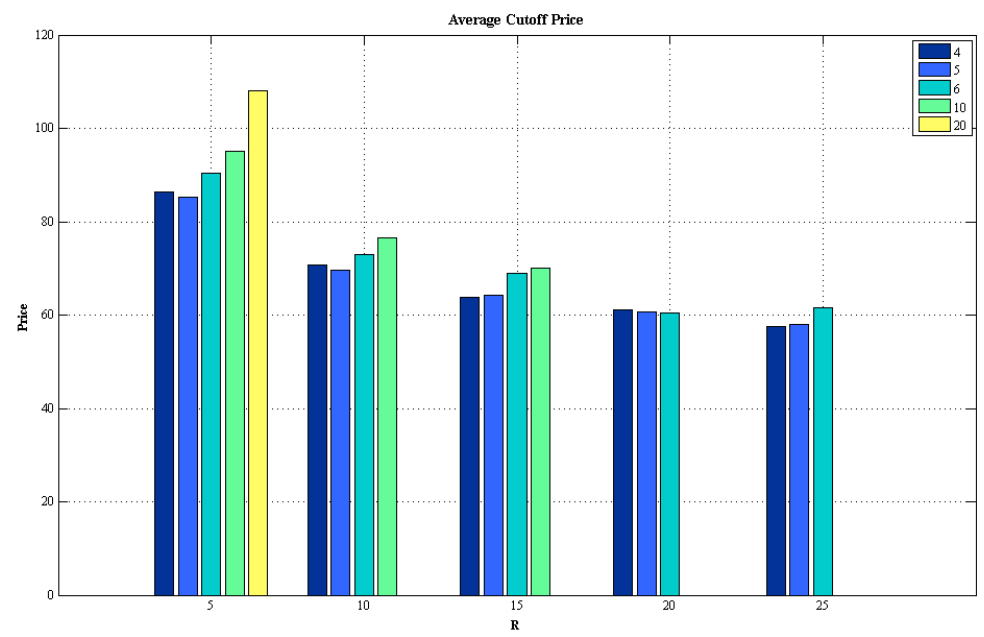

Figure 3: Average cutoff price results for Scenario 1

\section{Percentage of assigned spectrum}

As the supply of spectrum increases in the market, there is less efficiency in the assignment of spectrum. For this particular parameter, our best results are obtained when $\mathrm{R}=5$ and there are 20 spectrum users with an $85.7 \%$ of the spectrum being assigned. The worst case correspond to the situation where $\mathrm{R}=25$ and there are only 4 spectrum users, which results in an assignment of the $43.4 \%$ of the spectrum. It should be noted that given the particular requirements for spectrum assignment inherent to the LTE standard, in situations where there is spectrum under supply, we can observe that the resulting assignment is not completely smooth across the distribution of spectrum users. This irregularity disappears as the spectrum availability increases in the market.

Taking into account our aggregate data, we have determined that the average percentage of assigned spectrum in this scenario is $61 \%$. This is the value that we have considered as our threshold for the calculation of the final market viability score. 


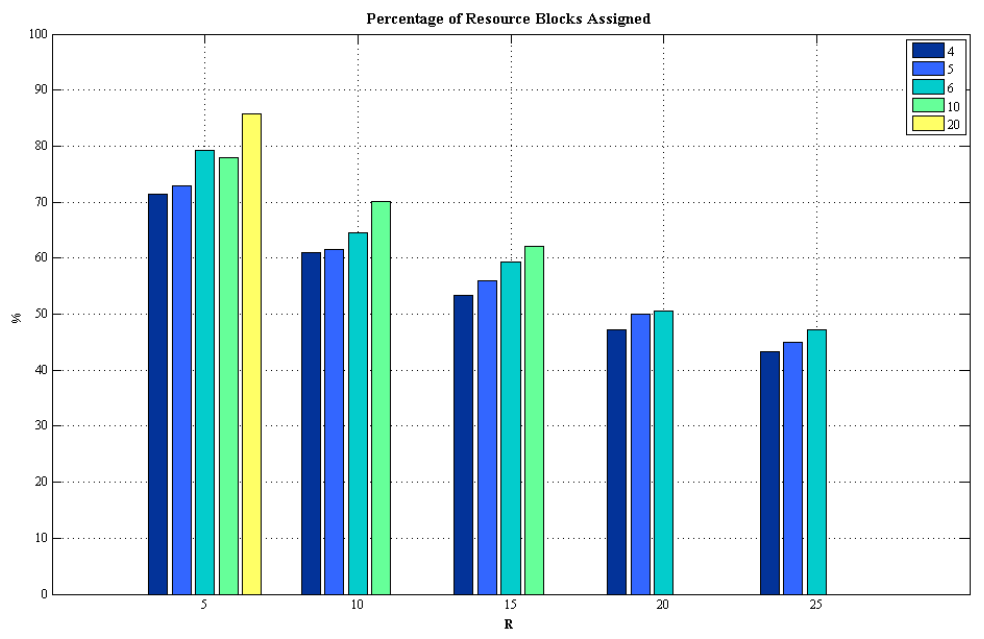

Figure 4: Percentage of assigned Resource Blocks in Scenario 1

\section{Average number of unlicensed spectrum units per spectrum user}

It follows intuition that as spectrum supply increases, spectrum users will have higher opportunities to obtain licensed RBs from the market, and thus, they will opt for less unlicensed spectrum units (if at all). This can be supported by the results presented in figure 5 . As it can be observed, in scenarios of spectrum oversupply, spectrum users will hold on to 1.3 unlicensed bandwidth units on average, while on the case of spectrum under supply, this value will be slightly above 4 . We should remember that given the characteristics of our model, spectrum users need on average 4 unlicensed units to fulfill their traffic requirements. Hence, it is important to mention, that even if there is a notorious difference between our best and worst cases, spectrum users in this scenario are not accumulating unlicensed units above their average unlicensed spectrum requirement. 


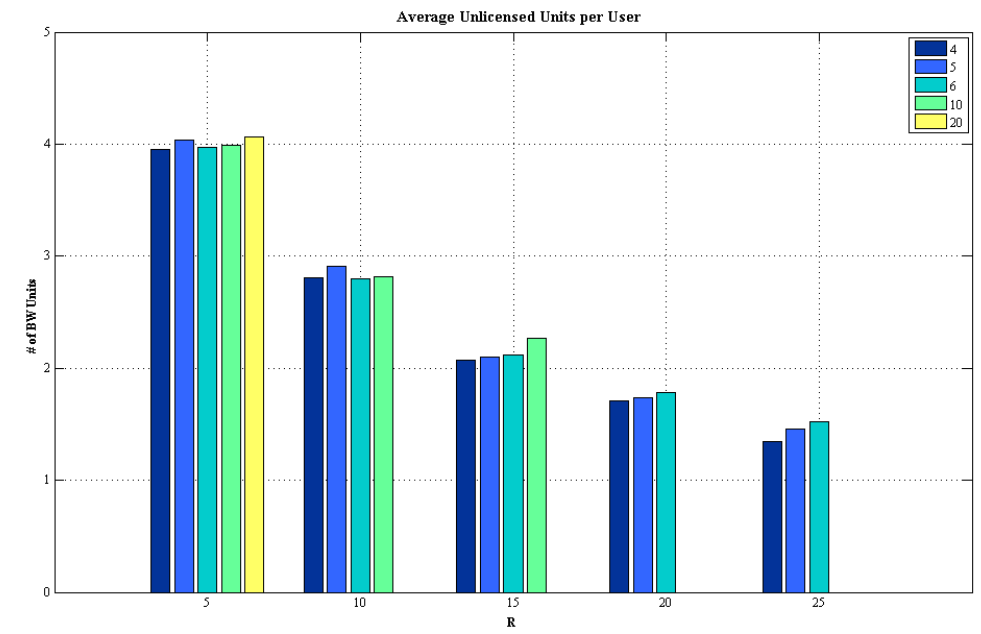

Figure 5: Average number of unlicensed spectrum bandwidth units per spectrum user in Scenario 1

\section{Final Market Viability Score for Scenario 1}

After evaluating the data we obtained via simulations with the criteria presented in 3, we obtained the final Market Viability Score for this first scenario, which is presented in figure 6 .

Our first goal with this calculation is to determine the feasibility of a market system carried out under virtualization concepts, and at the same time, contrast this values with those obtained with the first version of SPECTRAD, (presented in [11]), in order to find out whether there is improvement in the market liquidity conditions. 


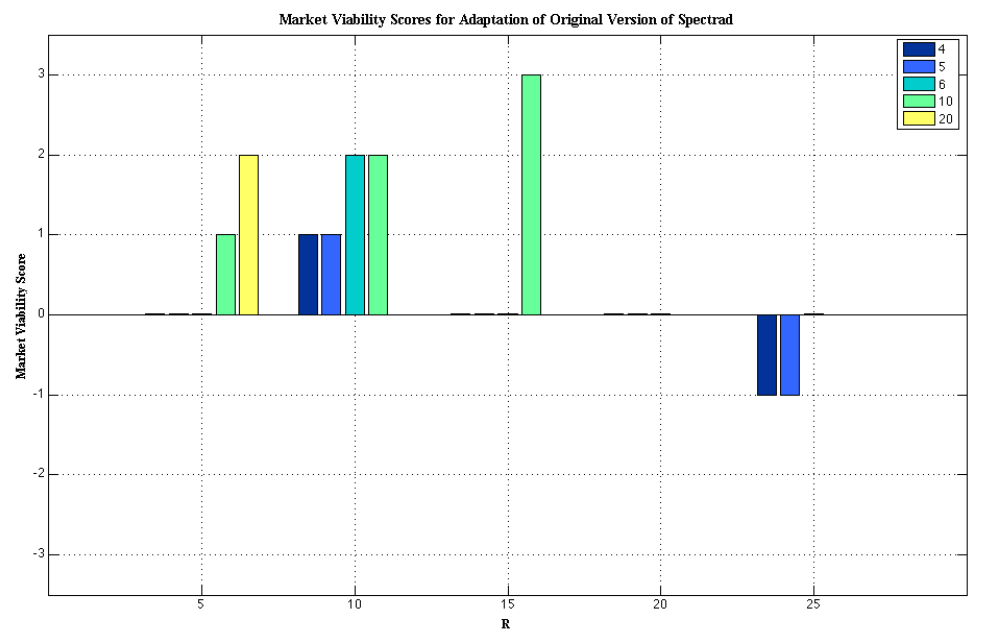

Figure 6: Market Viability Score for Scenario 1

Our results present a degree of improvement from those obtained in [11] for the simulated markets, including a new viable market in the list. Nevertheless, we coincide with the original model on the situations in which viable markets can be achieved, which is when $\mathrm{R}=5, \mathrm{R}=10$ and $\mathrm{R}=15$. None of the aforementioned cases represent situations of spectrum oversupply. We should remember that in the case of $R=10$ and $R=15$, the maximum number of spectrum users we tested, given our spectrum availability settings, is 10. What should be remarked is that in our $\mathrm{R}=10$ case, all the combinations of users we tested resulted in a viable outcome.

\subsubsection{Scenario 2}

Once we have analyzed the results of our initial approach, we would like to explore the impact of further changes in our model in the final market liquidity. The main variation in this scenario corresponds to the duration of the usage of the unlicensed spectrum bandwidth units, which corresponds to the same amount of time as the licensed spectrum lease. We present them in the following figures. Again, figures 7 - 10 show the detailed values obtained for each viability criteria, while figure 11 presents the final market viability score obtained in this new scenario. 


\section{Probability that demand is greater than supply}

In figure 7 we can observe a significant improvement in the values of the probability of having demand being greater than supply. In this particular scenario, the new (shorter) duration of unlicensed spectrum usage prompts spectrum users to be more active participants in the market, increasing the values associated with this factor. Indeed, when $\mathrm{R}=5$, for all the different user groups we have considered, the demand is always greater than the supply (100\% probability). The lowest value of this factor corresponds to $18.3 \%$, which represents the case where $\mathrm{R}=25$ and there are 4 spectrum users participating in the market (i.e., spectrum oversupply). It is important to point out that even under these oversupply conditions, the rate of this factor does not drop to extremely low values (i.e., $<1 \%$ ), which were considered as signs of lack of liquidity in the original model.

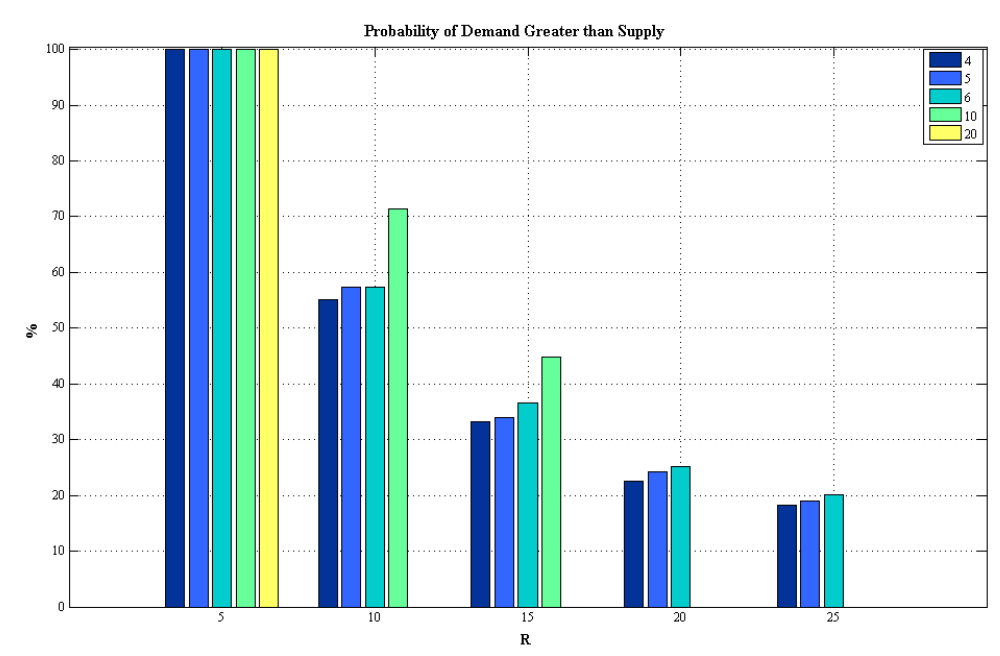

Figure 7: Probability that demand is greater than supply results for Scenario 2

\section{Average cutoff price}

The increase of the participation of spectrum users in the market has an important impact in the average cutoff price as well. Under this new model, the highest cutoff price rises up to 161.8 monetary units and it occurs under spectrum scarcity circumstances, specifically when $\mathrm{R}=5$ and there are 20 spectrum users in the market. The lowest cutoff price, 66.5 monetary units, is obtained in oversupply situations, when $R=25$ and only 4 spectrum users 
are participating in the market. All the results obtained for this factor can be observed in figure 8 .

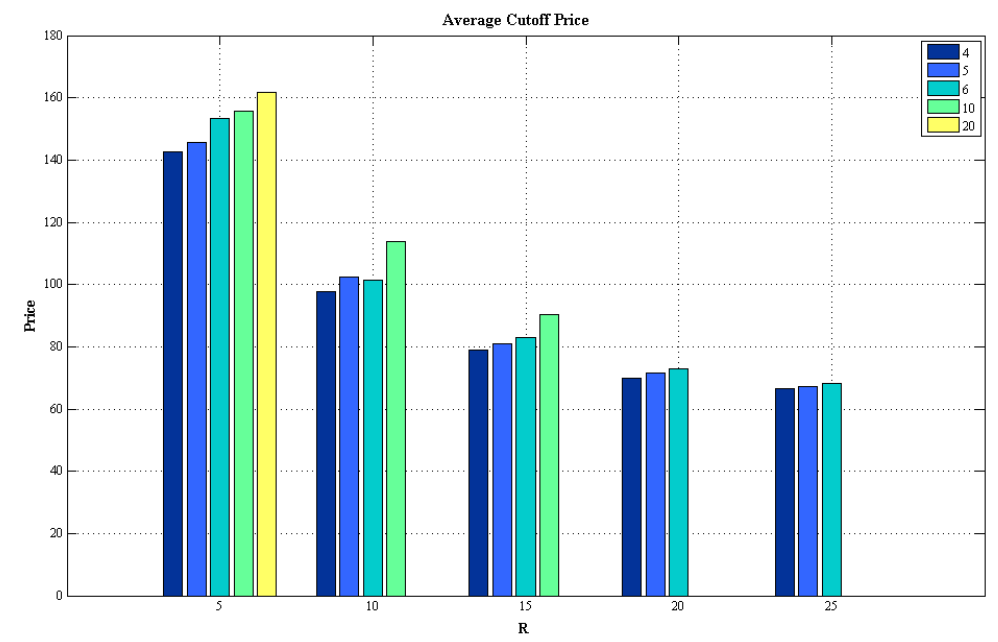

Figure 8: Average cutoff price results for Scenario 2

\section{Percentage of assigned spectrum}

As we had previously mentioned, the process of assigning spectrum becomes more complex when incorporating LTE into the model as we need to consider a set of rules inherent to the standard. For this specific reason, in situations when spectrum is scarce (e.g., $R=5$ ), the progression in spectrum assignment is not entirely smooth. This was already evidenced in our previous simulated scenario. In spite of this situation, we can observe an increase in the efficiency of spectrum assignment in this particular case. The highest percentage of RBs assigned is $99.2 \%$, which is achieved when $\mathrm{R}=5$, while the lowest is $52.1 \%$, achieved when $\mathrm{R}=25$. Figure 9 shows the values obtained for this parameter in the simulations performed for this second scenario.

Considering our aggregate data, we have estimated that the average percentage of assigned RBs, considering all of our performed simulations is $76 \%$. This is the threshold that we have considered for the estimation of the final market viability score, regarding this criterion, in this second scenario. 


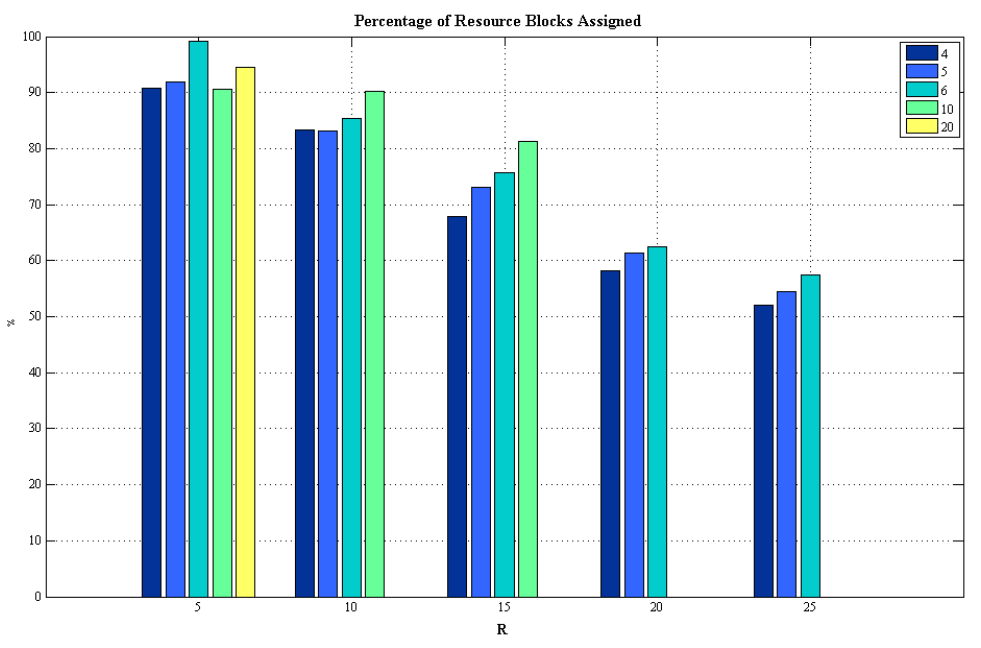

Figure 9: Percentage of assigned Resource Blocks in Scenario 2

\section{Average number of unlicensed spectrum units per spectrum user}

This factor is associated with the main variation we performed in this second scenario, thus, we expected to obtain a significant change in the resulting values. Indeed, as we can observe in figure 10, the maximum amount of unlicensed bandwidth units that have been accumulated on average by the spectrum users is approximately 2.3. This value progressively decreases until it reaches 0.37 in situations of spectrum oversupply. Even the maximum value obtained is well below the average number of unlicensed bandwidth units that spectrum users need to satisfy the traffic requirements in the market (4 Bandwidth units). 


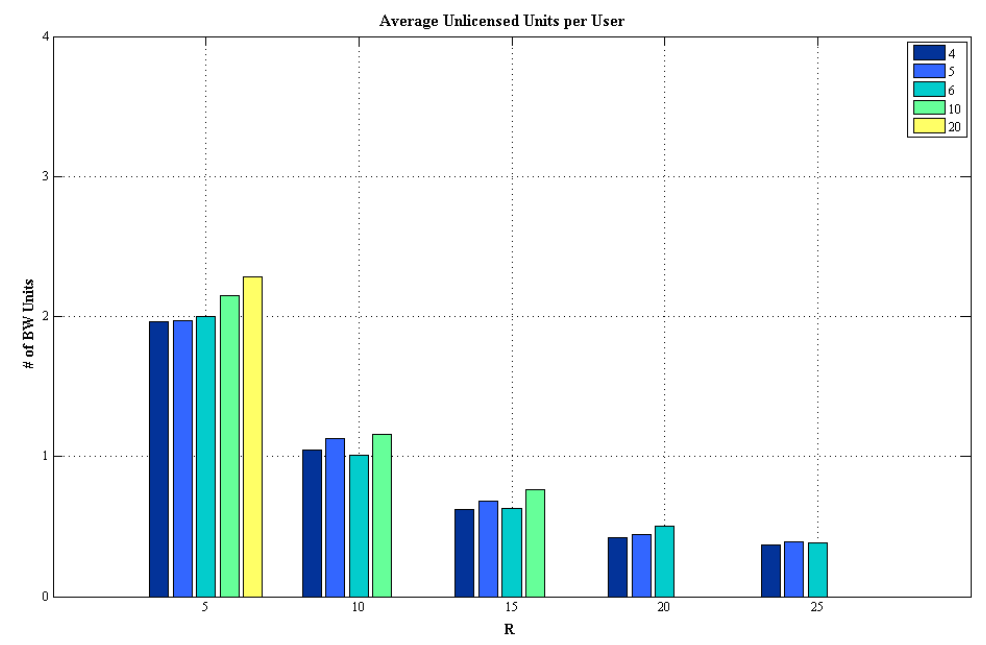

Figure 10: Average number of unlicensed spectrum bandwidth units per spectrum user in Scenario 2

\section{Probability of Empty Bid List}

In this scenario, the resulting probability of having an empty bid list is zero in all the cases that we have tested. This is the consequence of the limited duration of the usage of the unlicensed spectrum. Given that the licensed spectrum lease and the usage time of unlicensed spectrum last the same period (10 simulation time units), spectrum users are prompted to participate in the market and determine whether they are competitive enough to obtain licensed spectrum leases.

\section{Final Market Viability Score for Scenario 2}

After seeing significant improvements in the particular criteria for the market viability evaluation, we present in figure 11 the scores that we have obtained. As it can be observed, in this new scenario, we find positive outcomes for all the cases we have tested, including when we take into account spectrum under supply and oversupply conditions. Moreover, these favorable outcomes include the presence of a small number of market participants, which is the situation that most resembles our current telecommunications market structure. 


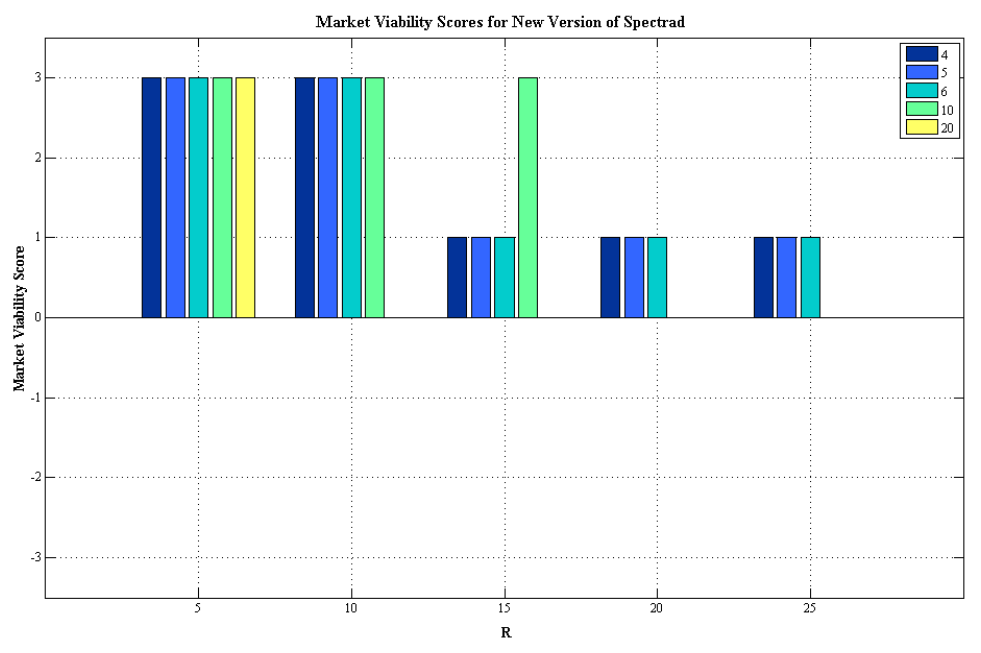

Figure 11: Market Viability Score for Scenario 2

\section{Discussion}

In order to discuss the results we presented in the previous section, we shall remember that the main reason why we decided to incorporate wireless networks virtualization notions in a spectrum trading model was to be able to find a trading commodity that would bring thickness to the market and hence would improve the overall market liquidity outcome.

In the first scenario, compared to the original version of SPECTRAD, we could only evidence a slight improvement in liquidity. Nevertheless, we should remember that in this particular case, our assumption of "perfect spectrum fungibility" is framed within an existing technology, which is LTE-A. Additionally, if we pay attention to particular details such as the probability that demand is greater than supply, we can already find significant improvements which are inherent to the implementation of the pool of LTE resource blocks in our model. We find that under the new circumstances, there is not a one-to-one mapping of capacity (i.e., one resource block represents a specific capacity value), instead, a group of resource blocks is associated with specific values of capacity, which in turn has its impact on the bids that users post, and consequently in the overall demand in the market.

The second scenario we presented, not only represents significant individual improvements for the market viability criteria, but also for the overall market 
scores. One of the main factors that contributes to this liquidity enhancement is the fact that the probability of having a bid list empty has decreased to zero for all the tested scenarios. This is a direct representative of the increased participation of the spectrum users in the market, which has further implications on the positive variations of the remaining viability criteria.

Figure 12 presents the market viability results obtained in the three versions of SPECTRAD that have been mentioned throughout our work. The leftmost graph corresponds to the original SPECTRAD model presented in [11], showing the values that are comparable to the model presented in this analysis. The center and rightmost figures correspond to the viability scores for Scenarios 1 and 2, which were already introduced in section 3. In this figure, we can visually appreciate the changes that the adoption of wireless virtualization has brought to our specific spectrum trading model.
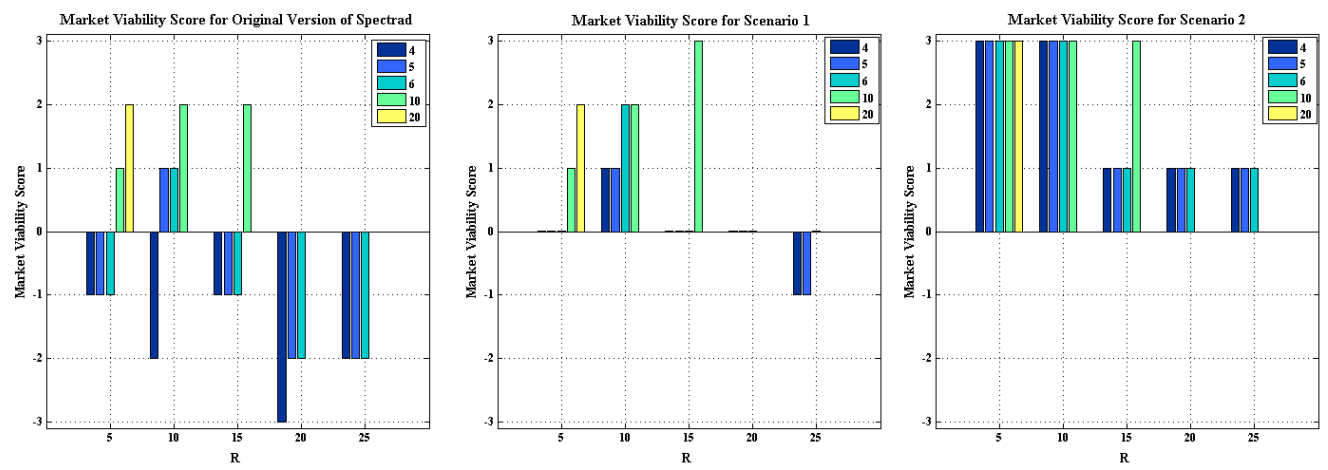

Figure 12: Comparison of the Market Viability Scores for different SPECTRAD Model Versions

\subsection{Additional Technical Details}

Regarding carrier aggregation, the current release of the LTE standard contemplates the association of only certain bands for the intra-band and interband deployment of this procedure. Nevertheless, for ease of implementation of our model, and given that we were already restricting it to a rather small set of available bands in the United States, we contemplated the fact that these three bands could be associated with each other for carrier aggregation purposes, even if the standard does not support this yet. The different parameters and specifications regarding carrier aggregation have significantly 
evolved in the past releases of the LTE standard, it is in this way that we have considered that the bands we have assumed to be compatible may be included in upcoming releases, thus supporting the validity of our analysis.

\section{Conclusions and Future Research}

Our current work represents our first step towards incorporating wireless virtualization concepts in a spectrum trading scenario. By introducing in the market, the notion of a pool of spectrum resources by taking advantage of the benefits of the LTE-standard, we have been able to provide further opportunities for spectrum users to opt for spectrum in the market. At the same time, this has permitted us to refine traded commodity, which has increased the scenarios in which we can find viable markets. We still consider, however, that our model can be further enhanced by delving deeper into features and advantages that are offered by (and can be exploited from) the LTE-A standard and additional virtualization nuances.

Another important step we would like to take is the diversification of the possibilities available to the market participants (spectrum users) in terms of the pool of spectrum resources. For instance, we would like the pool to cover spectrum belonging to the IEEE 802.11 standard in the $5 \mathrm{GHz}$ range, due to the considerable amount of bandwidth available in this band. In this way, we could explore the benefits and constraints of this spectrum diversification in the market behavior, and perhaps determine further realizable market scenarios.

\section{References}

[1] R. H. Coase, "The federal communications commission," Journal of law and economics, pp. 1-40, 1959.

[2] C. Caicedo and M. B. Weiss, "The viability of spectrum trading markets," IEEE Communications Magazine, vol. 43, no. 3, pp. 46-52, 2011.

[3] M. M. Gomez and M. B. Weiss, "How do limitations in spectrum fungibility impact spectrum trading?" Telecommunications Policy Research Conference, September 2013. 
[4] L. Doyle, J. Kibilda, T. K. Forde, and L. DaSilva, "Spectrum without bounds, networks without borders," Proceedings of the IEEE, vol. 102, no. 3, pp. 351-365, March 2014.

[5] C. E. Caicedo, "Technical architectures and economic conditions for viable spectrum trading markets," Ph.D. dissertation, University of Pittsburgh, 2009.

[6] J. W. Mayo and S. Wallsten, "Enabling efficient wireless communications: The role of secondary spectrum markets." Information Economics and Policy, vol. 22, no. 1, pp. 61-72, 2010.

[7] G. R. Faulhaber and D. J. Farber, Rethinking rights and regulations: institutional responses to new communication technologies. MIT Press, 2003, ch. Spectrum management: Property rights, markets, and the commons., pp. 193-226.

[8] M. Bykowsky, "A secondary market for the trading of spectrum: promoting market liquidity." Telecommunications Policy, vol. 27, no. 7, pp. 533-541, 2003.

[9] P. Crocioni, "Is allowing trading enough? making secondary markets in spectrum work." Telecommunications Policy, vol. 33, no. 8, pp. 451-468, 2009.

[10] R. Matheson and A. C. Morris, "The technical basis for spectrum rights: Policies to enhance market efficiency," Telecommunications Policy, vol. 36, no. 9, pp. 783-792, October 2012.

[11] C. E. Caicedo and M. B. Weiss, "The viability of spectrum trading markets." New Frontiers in Dynamic Spectrum, 2010 IEEE Symposium on, April 2010.

[12] T. K. Forde, I. Macaluso, and L. E. Doyle, "Exclusive sharing and virtualization of the cellular network," New Frontiers in Dynamic Spectrum Access Networks (DySPAN), 2011 IEEE Symposium on., 2011.

[13] A. Wang, M. Iyer, R. Dutta, G. N. Rouskas, and I. Baldine, "Network virtualization: Technologies, perspectives, and frontiers." Lightwave Technology, Journal of, vol. 31, no. 4, pp. 523-537, 2013. 
[14] Y. Zaki, L. Zhao, C. Goerg, and A. Timm-Giel, "Lte mobile network virtualization," Mobile Networks and Applications, vol. 16, no. 4, pp. 424-432, 2011.

[15] T. A. Weiss and F. K. Jondral., "Spectrum pooling: an innovative strategy for the enhancement of spectrum efficiency," IEEE Communications Magazine, vol. 42, no. 3, pp. S8-14, 2004.

[16] S. Hua, P. Liu, and S. S. Panwar, "The urge to merge: When cellular service providers pool capacity." IEEE International Conference on Communications, 2012.

[17] T. Forde and L. E. Doyle, "Cellular clouds," Telecommunications Policy, vol. 37, no. 2-3, pp. 194-207, 2013.

[18] S. Ahmadi, LTE-Advanced: A Practical Systems Approach to Understanding 3GPP LTE Releases 10 and 11 Radio Access Technologies. Academic Press, 2013.

[19] A. Ghosh, J. Zhang, J. G. Andrews, and R. Muhamed, Fundamentals of LTE. Pearson Education, 2010.

[20] 3GPP, "Evolved universal terrestrial radio access (e-utra); base station (bs) radio transmission and reception (3gpp ts 36.104 version 12.3 .0 release 12)," 3GPP, TS 12.3.0:V12.3.0, March 2014.

[21] ETSI and LTE, "Requirements for further advancements for evolved universal terrestrial radio access (e-utra) (lte-advanced)(3gpp tr 36.913 version 11.0.0 release 11)," ETSI, TR 136.913:V11.0.0, November 2012. 